\title{
Assessment of dynamic behaviour of earth dams taking into account large strains
}

\author{
Mirziyod Mirsaidov", Tokhirjon Sultanov, Javlon Yarashov, Elyor Toshmatov \\ Department of Theoretical and Constructional Mechanics, Tashkent Institute of Irrigation and \\ Agricultural Mechanization Engineers, 39 KoriNiyoziy str., Tashkent 100000, Uzbekistan
}

\begin{abstract}
This paper presents the results of the assessment and prediction of the dynamic behavior of earth dams of various heights, taking into account large strains (geometric nonlinearity) and inhomogeneous features of structures under multi-component kinematic effects. A mathematical model, methodology and algorithm for estimating dynamic behavior of earth dams taking into account inhomogeneous features of structures and large strains in spatial and plane statements are given. Dynamic behavior of the models of the Pachkamar, Gissarak and Nurek earth dams has been studied taking into account large strains and inhomogeneous features of structures in various pre-resonant and resonant modes under multicomponent kinematic effect. A number of new effects associated with the manifestation of large strains in the structure under various dynamic influences are revealed.
\end{abstract}

\section{Introduction}

Large-scale hydro-engineering construction is associated with the construction and operation of large dams and reservoirs, which can not only significantly change the conditions of ecosystems, socium, physical and geographical characteristics of entire region, but also pose a potential risk of major accidents and man-made emergencies as a result of failures and malfunctions of hydrotechnical structures.

In the Central Asian region, of the greatest concern are the conditions of more than 300 dams aimed to regulation the water flow on transboundary rivers. Dams and other waterworks, built more than 30-40 years ago, due to their aging and reduced quality of operation, pose a serious danger to the population and facilities located in developed areas downstream the dams. Therefore, the compliance with design requirements, maintenance of hydrotechnical structures in good condition, ensuring the required level of reliability and maintainability is of paramount importance [1].

Over the past 70 years, more than a thousand accidents in large hydrotechnical structures have occurred in the world, the main causes of which are: cracks, destruction of slopes, settlement of the foundation of earth dams and insufficient discharge capacity of spillway structures $[2,3]$.

\footnotetext{
* Corresponding author: theormir@mail.ru
} 
World experience shows that timely prevention is much more economical and more effective than the elimination of the consequences associated with flood events and accidents on earth hydrotechnical structures. Therefore, the organization of monitoring and forecasting of possible emergency situations, the implementation of protective engineering and technical measures to increase the stability of earth dams come to the fore.

Not all the issues of soil behavior under load are clarified to the end. On this account there are many different theories, more or less difficult to implement in solving specific problems. One of the important problems here is the consideration of non-linear strain in structures, in particular, large strains (i.e., geometric non-linearity). The need to account for geometrically non-linear strain of earth structures is increasing due to the construction of high earth structures. The question of how much this factor affects the stress-strain state (SSS), strength of structures and stability of slopes remains insufficiently studied and requires extensive research [4-9].

Dynamic strength of earth structures depends on the design features of the structure, its actual strain, geological conditions of the area of the site, and the distance from the epicenter of the earthquake.

Approximate assessment of the stress-strain state of structures in the process of design does not take into account the real nature of the structures, contribution of high vibration modes, non-linear strain of structure material and multi-component character of seismic impact. As the results of study of seismic event aftermath [4- 9] and theoretical studies in the field of seismic resistance of structures show, the aspects mentioned above are the causes of damage.

Along with this, there are a number of publications where a review on some specific issues related to the problem under consideration is covered [10-12].

Therefore, the assessment of strength and dynamics of earth structures should be carried out taking into account the inhomogeneous design features of structures, non-linear strain of structures and material under multicomponent dynamic effect, including resonant modes of oscillation.

\section{Method}

To predict the dynamics of earth dams in a three-dimensional statement, a spatial model of the structure is presented, as an inhomogeneous system (figure 1).

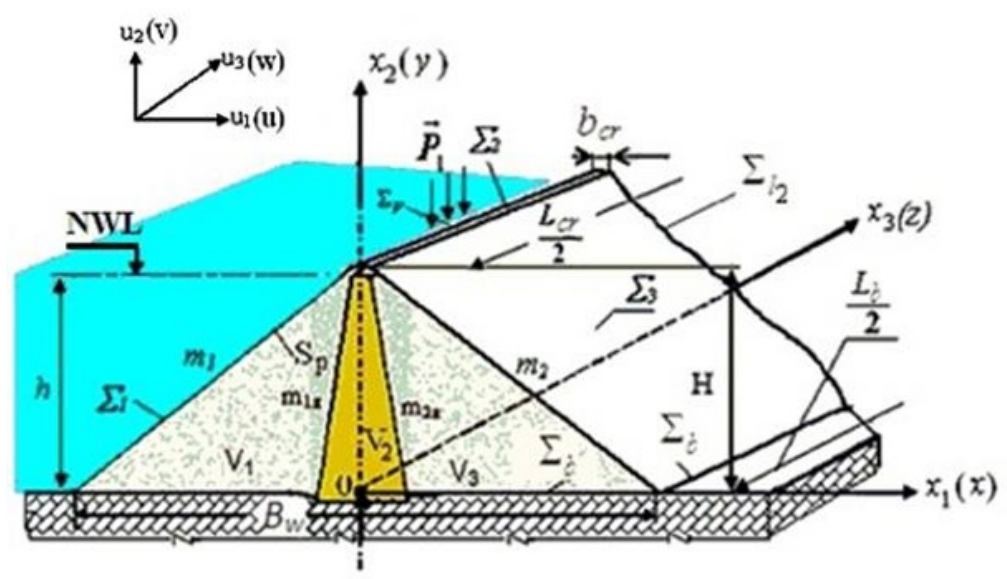

Fig.1. Design scheme of inhomogeneous three-dimensional system. 
The surfaces of the foundation and side slopes $\Sigma_{b}, \Sigma_{l_{1}}, \Sigma_{l_{2}}$ are rigidly fixed, the surface of the downstream slope $\Sigma_{3}$ is stress-free, water pressure acts on the surface Sp (the part of the upstream slope below the Normal water level (NWL)), and an external load $\Sigma_{2}$ is applied on the crest surface $\Sigma_{p}$.

Here: $V=V_{1}+V_{2}+V_{3}$ is the volume of the dam body $\left(V_{1}, V_{3}\right.$ are the volumes of the upper and lower retaining prisms, $V_{2}$ is the volume of the core); $\sum_{l_{1}}, \Sigma_{l_{2}}$ are the surfaces of the bank slopes, $\sum_{b}$ is the surface of the foundation along the bottom, $\Sigma_{1}, \sum_{2}, \sum_{3}$ are the surfaces of the retaining prisms and the crest.

To simulate the process of strain and the dynamics of earth dams (figure 1) in spatial statement, the Lagrange variation equation is used, based on the d'Alembert principle, for inhomogeneous deformable three-dimensional bodies:

$$
\begin{gathered}
-\int_{V_{1}} \sigma_{i j} \delta \varepsilon_{i j} d V-\int_{V_{2}} \sigma_{i j} \delta \varepsilon_{i j} d V-\int_{V_{3}} \sigma_{i j} \delta \varepsilon_{i j} d V- \\
-\int_{V_{1}} \rho_{1} \ddot{\vec{u}} \delta \vec{u} d V-\int_{V_{2}} \rho_{2} \ddot{\vec{u}} \delta \vec{u} d V-\int_{V_{3}} \rho_{3} \ddot{\vec{u}} \delta \vec{u} d V+ \\
+\int_{V} \vec{f} \delta \vec{u} d V+\int_{S_{P}} \vec{p} \delta \vec{u} d S+\int_{\Sigma_{P}} \vec{P} \delta \vec{u} d \Sigma=0, \\
i, j=1,2,3 .
\end{gathered}
$$

When solving the problems, physical properties of the body are described by the relations between the components of the stress $\sigma_{i j}$ and strain $\varepsilon_{i j}$ tensor with the generalized Hooke law:

$$
\sigma_{i j}=\lambda_{n} \varepsilon_{k k} \delta_{i j}+2 \mu_{n} \varepsilon_{i j}
$$

Here $\vec{u}, \varepsilon_{i j}, \sigma_{i j}$ - are the displacement vector, the strain and stress tensors, respectively; $\delta \vec{u}, \delta \varepsilon_{i j}$ - are the isochronous variations of displacements and strains; $\lambda_{n}$ and $\mu_{n}$ are the Lame constants; $\rho_{n}$ is the density of material of the system elements (the index $n=1,2,3$ means the part of the system to which this quantity relates); $\vec{f}$ is the vector of mass forces; $\vec{P}_{1}$ is the vector of external forces applied to the area $\Sigma_{\mathrm{p}} ; \vec{p}$ is the water pressure (the sum of hydrodynamic and hydrostatic pressures) resulting from the interaction of a structure with water medium and determined at a point $\left(x_{1}, x_{2}\right)$ by formula [7]

$$
\vec{p}\left(x_{1}, x_{2}, t\right)=\vec{p}_{\partial}+\vec{p}_{C}=-\rho_{0} \frac{\partial \varphi^{*}\left(x_{1}, x_{2}, t\right)}{\partial t}+\rho_{0} g\left(h-x_{2}\right),
$$

Where $\rho_{0}$ is the water density; (h- $\left.\mathrm{x}_{2}\right)$ is the depth of the point on the pressure face of the dam; $\varphi^{*}\left(x_{1}, x_{2}, t\right)$ is the velocity potential. 
To take into account large strains (i.e., geometric nonlinearity), in solving specific problems, the relation between the components of the strain tensor $\varepsilon_{i j}$ and the components of the displacement vector $\vec{u}=\left\{u_{1}, u_{2}, u_{3}\right\}$ is used:

$$
\varepsilon_{i j}=\frac{1}{2}\left(u_{i, j}+u_{j, i}+u_{\ell, i} \cdot u_{\ell, j}\right) ; i, j, \ell=1,2,3
$$

Here along with the linear derivative of displacements from the coordinates, their quadratic components are also involved.

In all the problems considered, the displacement vector in the spatial coordinate system $\vec{x}=\left\{x_{1}, x_{2}, x_{3}\right\}=\{x, y, z\} \quad$ has three components of displacement $\vec{u}=\left\{u_{1}, u_{2}, u_{3}\right\}=\{u, \mathrm{v}, \mathrm{w}\}$.

Further, when creating mathematical models, the following conditions are taken into account:

- when solving dynamic problems, kinematic boundary conditions are taken into account at the foundation of the structure

$$
\vec{x} \in \sum_{0}: \vec{u}_{0}(\vec{x}, t)=\vec{\psi}_{1}(t)
$$

Boundary conditions at $\mathrm{t}=0$ :

$$
\vec{x} \in V: \vec{u}(\vec{x}, 0)=\vec{\psi}_{2}(\vec{x}) ; \dot{\vec{u}}(\vec{x}, 0)=\vec{\psi}_{3}(\vec{x})
$$

Here $\vec{\psi}_{1}(t)$ is the arbitrary time function, $\vec{\psi}_{2}(\vec{x}), \vec{\psi}_{3}(\vec{x})$ are the given functions of coordinates.

Now the general variation problem can be formulated as follows: it is necessary to determine the fields of displacements $\vec{u}(\vec{x}, t)$, strains $\varepsilon_{i j}(\vec{x}, t)$ and stresses $\sigma_{i j}(\vec{x}, t)$ in a nonlinear inhomogeneous spatial system (figure 1) arising under the action of mass forces $(\vec{f})$, external forces $\left(\vec{P}_{1}\right)$, inertia forces, and water pressure (3), which satisfy equations (1) - (2), (4) and the corresponding kinematic (5) and initial conditions (6) at any possible displacement $\delta \vec{u}$.

In the study of unsteady forced oscillations of a structure, the solution of the variation equation (1), with account of boundary (4) and initial conditions (5), is sought in the form:

$$
\vec{u}(\vec{x}, t)=\vec{u}_{0}(\vec{x}, t)+u^{*}(\vec{x}, t)
$$

Here $\vec{u}^{*}(\vec{x}, t)$ is the vector of sought for displacements; $\vec{u}_{0}(\vec{x}, t)$ is the given kinematic effect.

\subsection{Inhomogeneous linear system (figure 1)}

Substitution of expression (7) into (1) and the subsequent use of the finite element method (FEM) procedure reduces the problem under consideration to solving a system of ordinary differential equations of the $\mathrm{N}$-th order

$$
\begin{gathered}
{[M]\{\ddot{u}\}+[C]\{\dot{u}\}+[K]\{u\}=\{F\}+\{f(t)\}} \\
\text { or } \quad[M]\{\ddot{u}\}+2 \varepsilon[M]\{\dot{u}\}+[K]\{u\}=\{F\}+\{f(t)\}
\end{gathered}
$$

At initial conditions 


$$
\{u(0)\}=\left\{u_{0}\right\},\{\dot{u}(0)\}=\left\{\mathrm{v}_{0}\right\} .
$$

In the three-dimensional case, an isoparametric finite element in the form of a parallelepiped with 24 degrees of freedom is used for discretization of the system under consideration, and in a plane case - a triangular finite element with 6 degrees of freedom [13].

Here $[K],[M]$ are the stiffness and mass matrices of the system under consideration; $[\mathrm{C}]$ is the matrix of dissipative forces, taken as $[\mathrm{C}]=2 \varepsilon[\mathrm{M}]$, according to [14]; $\{u(t)\}$ is the vector of the sought for displacement amplitudes; $\{f(t)\}$ is the vector of external load from kinematic impact; $\{F\}$ is the total vector of external loads (mass forces, hydrostatic pressure of water, etc.); $\varepsilon=\frac{\delta}{2 \pi} \omega$ is the double coefficient of oscillation damping; $\delta$ is the logarithmic decrement of oscillations; $\omega$ is the natural frequencies of the structure. Equation (8) at initial conditions (9) is solved by direct integration using the Newmark method [15].

\subsection{Inhomogeneous system (figure 1) taking into account large strains}

The considered variation problem (1) after substituting expression (7) into (1) and finiteelement discretization is reduced to solving a system of nonlinear differential equations of $\mathrm{N}$-th order

$$
[M]\{\dot{u}\}+[C]\{\dot{u}\}+\mid K^{*}(u)\{u\}=\{F\}+\{f(t)\}
$$

At initial conditions (9).

Here, the elements of the matrix $\left.\mid K^{*}(u)\right\rfloor$ depend both on geometrical parameters of the structures and on its stress-strain state, expressed through nodal displacements; $[\mathrm{M}]$ is the mass matrix of the system under consideration; $[C]$ is the matrix of dissipative forces, if taken into account; $\{f(t)\}$ is the vector of external load from kinematic impact; $\{F\}$ is the total vector of external loads (mass forces, hydrostatic pressure of water, etc.).

Equation (9) at initial conditions (8) is solved by the direct integration method using the Newmark method [15]. To do this, at each time step $\Delta t$, the problem (10) - (9) is reduced to solving a system of algebraic equations of high order

$$
\begin{gathered}
{\left[A\left(u_{i}\right)\right]\left\{u_{i+1}\right\}=\left\{R_{i+1}\right\},} \\
{\left[A\left(u_{i}\right)\right]=\left[K\left(u_{i}\right)\right]+\frac{1}{\alpha t^{2}}[M]+\frac{\beta}{\alpha t}[C] .}
\end{gathered}
$$

The right-hand part $\left\{R_{i+1}\right\}$ of the equation (11) is determined by the expression

$$
\begin{aligned}
\{\mathrm{Ri}+1\} & =\{\mathrm{Pi}+1\}+[\mathrm{M}]\left(\frac{1}{\alpha \Delta t^{2}}\left\{u_{i}\right\}+\frac{1}{\alpha \Delta t}\left\{\dot{u}_{i}\right\}+\left(\frac{1}{2 \alpha}-1\right)\left\{\ddot{u}_{i}\right\}\right)+ \\
& +[C]\left(\frac{\beta}{\alpha \Delta t}\left\{u_{i}\right\}+\left(\frac{\beta}{\alpha}-1\right)\left\{\dot{u}_{i}\right\}+\frac{\Delta t}{2}\left(\frac{\beta}{\alpha}-2\right)\left\{\ddot{u}_{i}\right\}\right) .
\end{aligned}
$$


Thus, the algorithm for solving nonlinear differential equations (10) - (9) is as follows:

1. Initial values are set $\left\{u_{0}\right\},\left\{\dot{u}_{0}\right\},\left\{\ddot{u}_{0}\right\}=0$.

2. A system of algebraic equations (11) is formed, with the right-hand side of (13). Here, the elements of the matrix $\left[A\left(u_{i}\right)\right]$ are the functions of the strained state of the structure reached at the previous step.

3. The obtained system of algebraic equations (10) is solved by the Gauss method or the method of square root.

When solving specific problems, the order of solved systems of equations (10) with initial conditions (9) may reach more than 2000.

\section{Results}

Results of studies of the dynamics of various earth dams in a plane statement are given below.

The unsteady forced oscillations of the models of Nurek (height $\mathrm{H}=296 \mathrm{~m}$ ), Gissarak (height $\mathrm{H}=138 \mathrm{~m}$ ) and Pachkamar (height $\mathrm{H}=70 \mathrm{~m}$ ) earth dams [16, 17] have been investigated taking into account inhomogeneous features of structures under twocomponent kinematic effect in the foundation of the structures i.e.:

Horizontal effect

$$
\left\{u_{0}(t)\right\}=\left\{\begin{array}{lr}
a \sin (p t), & 0 \prec t \leq t^{*} \\
0, & t^{*} \succ t
\end{array}\right.
$$

Vertical effect

$$
\left\{v_{0}(t)\right\}=\left\{\begin{array}{lc}
b \sin (p t), & 0 \prec t \leq t^{*} \\
0, & t^{*} \succ t .
\end{array}\right.
$$

Below are the results obtained for the Gissarak dam at a frequency of the effect of $p=5.7 \mathrm{rad} / \mathrm{s}$; for Pachkamar dam at $p=6.85 \mathrm{rad} / \mathrm{s}$, for Nurek dam - at a frequency of the effect $p=4.5 \mathrm{rad} / \mathrm{s}$ and $p \approx \omega_{1}=5.07 \mathrm{rad} / \mathrm{s}$ ( $\omega_{1}$ is the first frequency of natural oscillations).

The impact amplitudes in all considered cases are $a=0.01 \mathrm{~m}, b=0.01 \mathrm{~m}$, and the duration of the kinematic action is $t^{*}=12 \mathrm{sec}$.

The energy dissipation in structure material is taken into account according to $[14,18]$ of the Sorokin model (proportional to stiffness) $[C]=\beta[K]$, with a coefficient $\beta=0.08$.

Figure 2 shows the displacements $\left(u_{1}, u_{2}\right)$ of a point on the crest of an inhomogeneous Pachkamar dam with coordinates $\left(x_{1}=-4.0 \mathrm{~m}, x_{2}=70.0 \mathrm{~m}\right)$, obtained taking into account large strains, i.e. geometrically nonlinear strain under two-component kinematic effect. 
a)

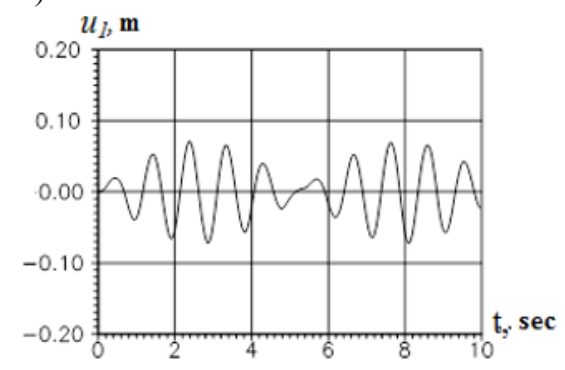

b)

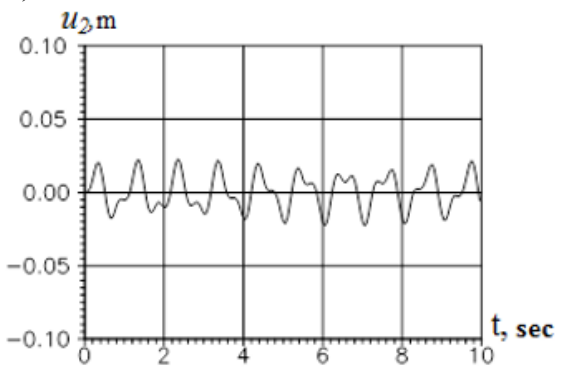

Fig.2. Change in displacements $u_{1}$ - (a) and $u_{2}$ - (b) of the point $\left(x_{1}=-4 \mathrm{~m}, x_{2}=70 \mathrm{~m}\right)$ of the Pachkamar dam under two-component kinematic effect

Analysis of the results obtained shows that for dams of average height the difference between linear displacements and displacements obtained taking into account geometric nonlinearity does not exceed $10 \%$. The greatest difference is manifested at the top and in the slope zones of the dam, and not immediately, but after some time from the beginning of the load; this is apparently due to a gradual increase in strain under dynamic effect.

Figure 4 shows the change over time of the difference between linear displacements $\bar{u}^{-l}$ and displacements $\bar{u}^{g n}$, taking into account the geometric nonlinearity of the point $\left(x_{1}=-\right.$ $\left.8.0 \mathrm{~m}, x_{2}=138.5 \mathrm{~m}\right)$ on the crest of an inhomogeneous Gissarak dam under two-component kinematic effect.

Analysis of the results (figure 3) shows that an account of geometric nonlinearity makes a significant correction (about 15\%) to the displacements of the high dam. The maximum difference is observed for the points of the upper part and the dam slopes some time after the start of the process. Unlike previous results, an essential difference is observed almost throughout the entire process of the effect. This once again confirms the conclusions that large strains in high structures under dynamic effect appear after its swinging and remain significant until the end of the effect.

a)

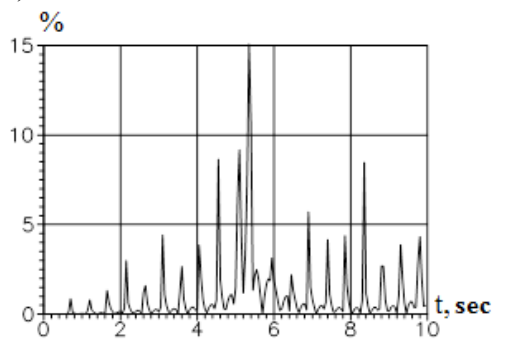

b)

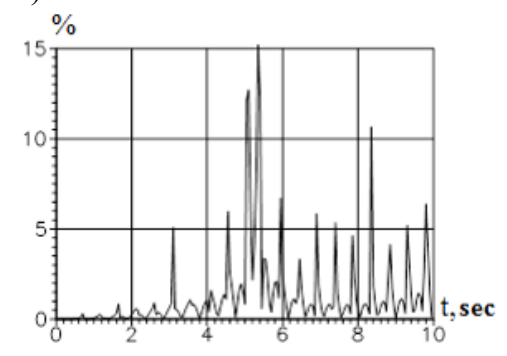

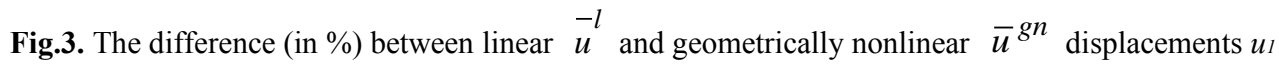
- (a) and $u_{2}$ - (b) of the point ( $\left.x_{1}=-8.0 \mathrm{~m}, x_{2}=138.5 \mathrm{~m}\right)$ on the crest of the Gissarak dam under twocomponent kinematic effect 
The forced nonlinear oscillations of the high Nurek dam in the pre-resonant (figure 4) and resonant (figure 5) modes have been investigated.

Figure 4 shows the time variation of the amplitude of stress intensity $\sigma_{i}$ at various points of the dam in the pre-resonant oscillation mode with the frequency of the effect $p=4.5 \mathrm{rad} / \mathrm{s}$ : for the point $\left(\mathrm{x}_{1}=-65.5 \mathrm{~m}, \mathrm{x}_{2}=265.2 \mathrm{~m}\right)-$ figure $4 \mathrm{a}$; for the point $\left(\mathrm{x}_{1}=-65.5 \mathrm{~m}\right.$, $\left.\mathrm{x}_{2}=228.2 \mathrm{~m}\right)-$ figure $4 \mathrm{~b}$; for the point $\quad\left(\mathrm{x}_{1}=-65.5 \mathrm{~m}, \mathrm{x}_{2}=80.2 \mathrm{~m}\right)-$ figure $4 \mathrm{c}$.

a)

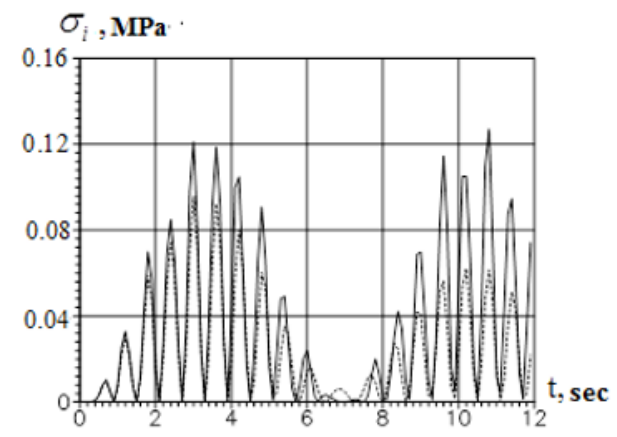

C)

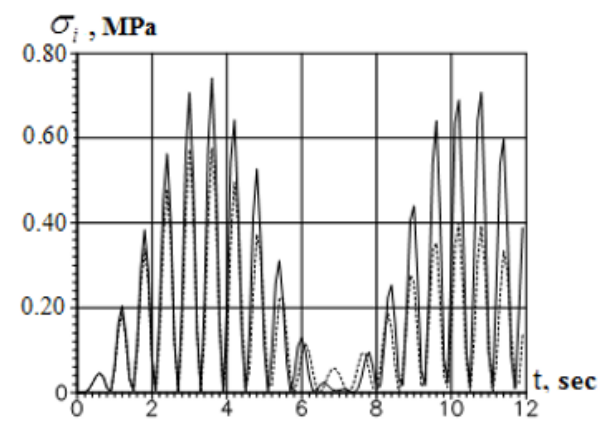

b)

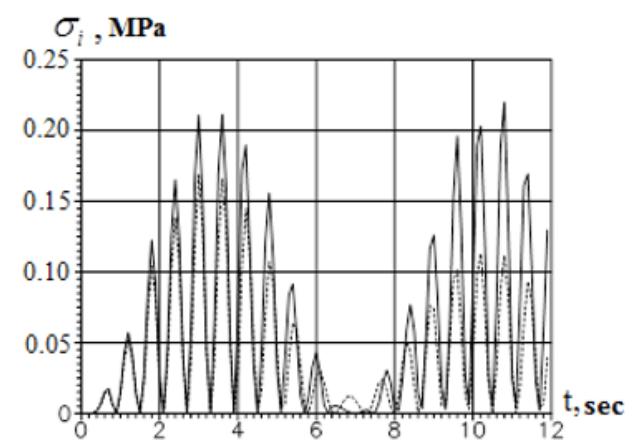

Fig.4. Change in stress intensity $\sigma_{i}$ in various points of the Nurek dam in the preresonant mode $(p=4.5 \mathrm{rad} / \mathrm{s})$ under twocomponent kinematic effect:

- - - - linear; ___ taking into account geometric nonlinearity

The results obtained, figure 4, demonstrate the oscillations of the Nurek dam, occurring in the "beat" mode. Such oscillations with two periods occur at two close (but not equal) carrier frequencies: frequencies of the effect are high-frequency oscillations with a small period, and frequencies of (basic) natural oscillations. The combination of these frequencies determines the pseudo-period — the time between oscillations with maximum amplitudes.

Figure 5 shows the change in the amplitude of the stress intensity $\sigma_{i}$ for the same points of the dam in the resonant mode, i.e. at kinematic effect with frequency $p \approx \omega_{1}=5.07$ $\mathrm{rad} / \mathrm{s}$ : figure $5 \mathrm{a}$ - for the point $\left(\mathrm{x}_{1}=-65.5 \mathrm{~m}, \mathrm{x}_{2}=265.2 \mathrm{~m}\right)$; figure $5 \mathrm{~b}-$ for the point $\left(\mathrm{x}_{1}=-65.5\right.$ $\left.\mathrm{m}, \mathrm{x}_{2}=228.2 \mathrm{~m}\right)$; figure $5 \mathrm{c}$ - for the point $\left(\mathrm{x}_{1}=-65.5 \mathrm{~m}, \mathrm{x}_{2}=80.2 \mathrm{~m}\right)$.

Isolines of stress intensity $\sigma_{i}$ in the cross section of a non-uniform Nurek dam at time $t$ $=10.2 \mathrm{sec}$ are shown in figure 6 : linear calculation - figure $6 \mathrm{a}$; calculation with account of geometric nonlinearity - figure $6 \mathrm{~b}$. 
The results obtained in figures 4-6 show the nature of a nonlinear effect, manifested in an increase in the amplitude of the stress intensity $\sigma_{i}$ at a constant oscillation period and a change in the stress state at fixed points in time.

a)

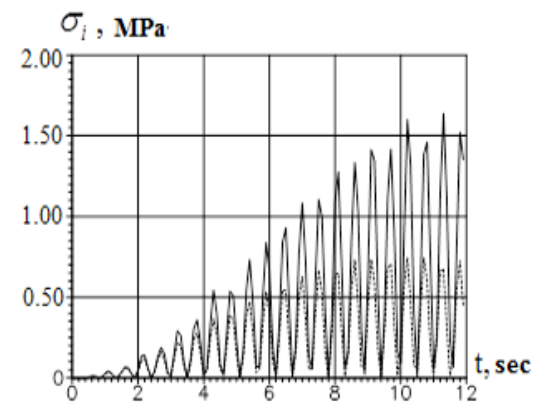

c)

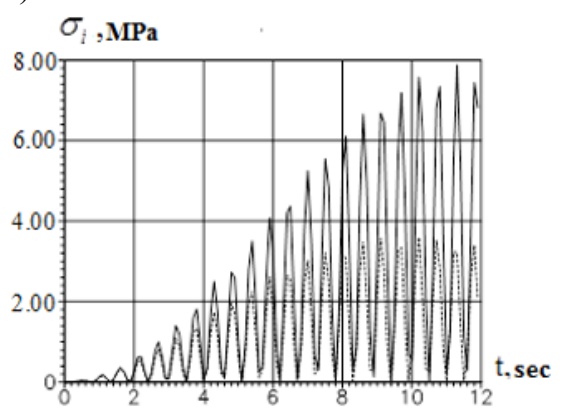

b)

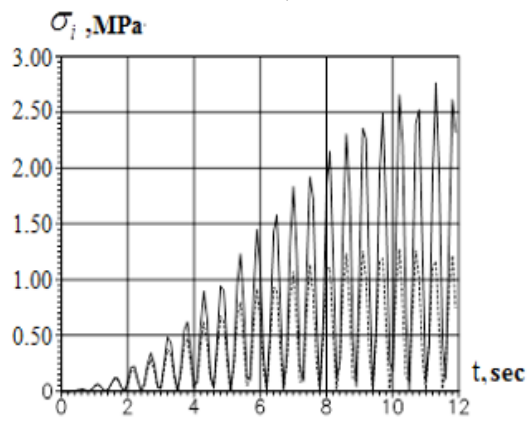

Fig.5. Change in stress intensity $\sigma_{i}$ at various points of the Nurek dam in the resonant mode under twocomponent effect with a frequency of

$$
p \approx \omega_{1}=5.07 \mathrm{rad} / \mathrm{s} \text { : }
$$

- - - - linear; taking into

account geometric nonlinearity

Calculations show that the geometric nonlinearity is most pronounced in the lower part of the dam, where the stress intensity $\sigma_{i}$ during the entire time of load effect is almost two times greater than the value obtained in the linear case.

a)

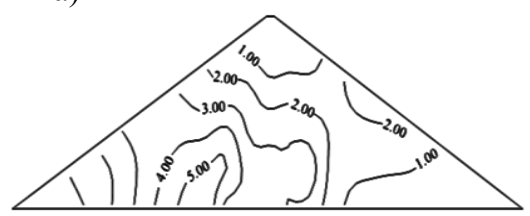

b)

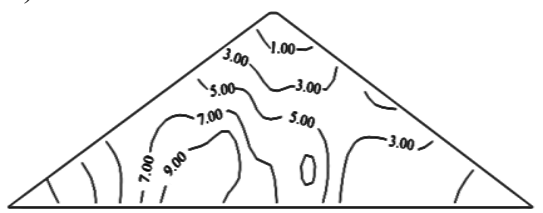

Fig.6. Isolines of stress intensity $\sigma_{i}(\mathrm{MPa})$ in the section of inhomogeneous Nurek dam in the resonant mode at time $t=10.2 \mathrm{sec}$

Studies carried out in the resonant mode of oscillations (figure 5) show an increase in the nonlinear effect of oscillations with an increase in the height of structures and the intensity of the effect.

\section{Discussion}

Analysis results of the dynamics and stress-strain state of earth dams of various heights, taking into account the geometric nonlinearity, make it possible to identify the dependence of the nonlinear effect on the size of the structure and its disposition, on duration and 
amplitude of the effect. At the same time, the proximity of the natural frequencies of the structure to the frequency of the effect (figure 5) has a significant effect on the manifestation of nonlinear effect. In the most unfavorable resonant mode, an account of large strains at some points in time leads to a significant increase in stresses in the lower part of a high structure. Accounting for geometric nonlinearity for inhomogeneous structures (figure 6) leads to a concentration of stresses at the contact of inhomogeneous parts of the structure.

\section{Conclusions}

A mathematical model, a method and an algorithm for estimating the dynamic behavior of earth dams taking into account large strains in a plane and spatial statements considering inhomogeneous features of structures are given in the paper.

Dynamic behavior of inhomogeneous earth dams of different heights is investigated.

It has been established that for dams of low and medium heights, consideration of geometrical nonlinearity does not lead to significant changes in the dynamics of structures as compared with the linear case.

For high dams, the difference in the dynamic behavior of earth dams under large strains taken into account, is significant, which mainly manifests itself after a certain time of the effect.

The greatest difference between the results of linear and nonlinear calculations is observed in the upper part and in the slope zones of the dam, when large strains are taken into account, and the nonlinear effect appears only after some time from the start of the effect; this is explained by the high rigidity of the dam, and, as a result, by the gradual increase in strains, in this case the consideration of geometric nonlinearity becomes essential.

Consideration of large strains and inhomogeneous structural features leads to stress concentration and growth at the contact of inhomogeneous parts of the structure.

The magnitude of the stress intensity $\sigma_{i}$, obtained taking into account the geometric nonlinearity, is almost two times greater than the values $\sigma_{i}$ obtained in a linear calculation, which is seen at sufficiently long effect. At the frequency of the effect close to the natural frequency of structure oscillations, this effect is enhanced and can lead to the destruction of structures.

\section{References}

1. E.Zh. Makhmudov. Proc. of the Republican Scientific-practical Conf. on Problems of reliability and Safety of Hydraulic Structures (Tashkent, Uzbekistan, 2006)

2. M.A. Akhmedov, K.D. Salyamova. Analysis and Assessment of Damage to Hydrotechnical Structures (Tashkent, Fan va tekhnologiya, 2016)

3. D.V. Stefanishin. News VNIIG named after B.E.Vedeneyev, 251 (2008)

4. N.D. Krasnikov. Seismic Stability of Hydrotechnical Structures Built of Earth Material (Moscow, Energoizdat, 1981) 
5. V.M. Lyatkher, I.N. Ivashenko. Earthquake Resistance of Seismic Dams (Moscow, Science, 1986)

6. Yu.K. Zaretsky, V.N Lombardo. Statics and Dynamics of Earth Dams (Moscow, Energoizdat, 1983

7. M.M. Mirsaidov, T.Z. Sultanov. Theory and Methods of Strength Assessment of Earth Dams (Saarbrucken, Lambert Academic Publishing, 2015)

8. K.S. Sultanov. J. Appl. Math. and Mech., 62(3) (1998)

9. K.S. Sultanov, B.E. Khusanov. Soil Mechanics and Foundation Engineering, 38(3) (2001)

10. M.M. Mirsaidov, T.Z. Sultanov, R.A. Abdikarimov. Magazine of Civil Engineering, 77(1) (2018).

11. M.M. Mirsaidov, T.Z. Sultanov. Magazine of Civil Engineering, 49(5) (2014)

12. M.M. Mirsaidov, T.Z. Sultanov, D.A. Khodzhaev. Assessment of the stress-strain state of earth dams. 8th Int. Conf. on Engineering Computational Technology (Dubrovnik, ECT, 2012)

13. T.Z. Sultanov, B.Sh. Yuldoshev, E.S. Toshmatov, J.A. Yarashov, R. Ergashev, M.M.Mirsaidov. MATEC Web of Conferences, 265 (2018)

14. V.T. Rasskazovskiy. Fundamentals of physical methods for determining seismic effects (Tashkent, Fan, 1973)

15. K. Bate, E. Wilson. Numerical methods of analysis and FEM (Moscow, Stroiizdat, 1982)

16. T.Z. Sultanov, D.A.Khodzhaev, M.M. Mirsaidov. Magazine of Civil Engineering, 45(1) (2014)

17. M.M. Mirsaidov, T.Z. Sultanov, Sh.A. Sadullaev. Magazine of Civil Engineering, 40(5) (2013)

18. M.M. Mirsaidov, T.Z.Sultanov. Soil Mechanics and Foundation Engineering, 49(6) (2013) 